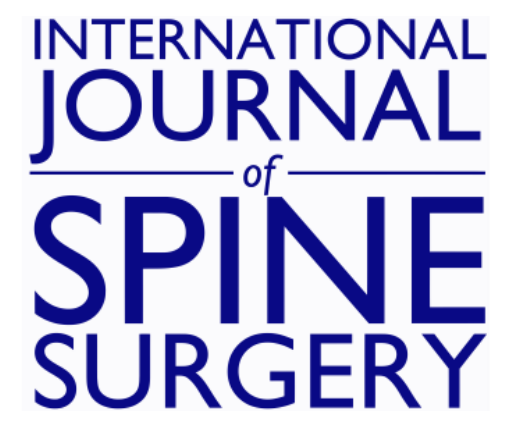

\title{
Thoracic epidural spinal angiolipoma with coexisting lumbar spinal stenosis: Case report and review of the literature
}

Mario Benvenutti-Regato, Rafael De la Garza-Ramos and Enrique Caro-Osorio

Int J Spine Surg 2015, 9 ()

doi: https://doi.org/10.14444/2067

http://ijssurgery.com/content/9/67

This information is current as of April 25, 2023.

Email Alerts Receive free email-alerts when new articles cite this article. Sign up at:

http://ijssurgery.com/alerts

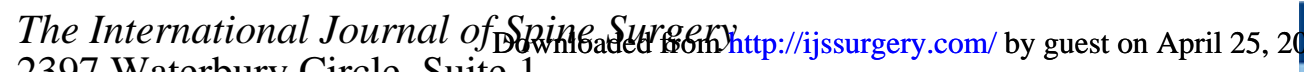
2397 Waterbury Circle, Suite 1,

Aurora, IL 60504, Phone: +1-630-375-1432

(C) 2015 ISASS. All Rights Reserved. 


\section{Thoracic epidural spinal angiolipoma with coexisting lumbar spinal stenosis: Case report and review of the literature}

Mario Benvenutti-Regato, MD,1,2 Rafael De la Garza-Ramos, MD,1,2 Enrique Caro-Osorio, MD 1,2

${ }_{1}^{1}$ Tecnológico de Monterrey, School of Medicine and Health Sciences, Monterrey, México ${ }^{2 N e u r o l o g y}$ and Neurosurgery Institute, Centro Médico Hospital Zambrano Hellion, TecSalud, Monterrey, México

\section{Abstract}

Background

Spinal angiolipomas (SALs) are uncommon benign lesions that may present insidiously with back pain or acutely with weakness due to tumor bleeding/thrombosis. Given their rarity, these lesions are often overlooked in the differential diagnosis of epidural masses. The purpose of this article is to report the case of an epidural SAL and to conduct a literature review on the topic.

Methods

A case report and review of the literature using the PubMed/Medline databases. All case reports and case series were reviewed up to June 2015.

Results

A 65-year old female presented with neurogenic claudication and magnetic resonance imaging (MRI) revealed lumbar spinal stenosis. Following decompressive surgery, she experienced symptom resolution, but three months postoperatively she presented to the emergency department with acute paraparesis. A thoracic MRI revealed a lesion located between $\mathrm{T} 8$ and $\mathrm{T} 10$ causing severe spinal cord compression. Following emergent laminectomy and en bloc resection, the patient regained function and the lesion was diagnosed as SAL. Our literature review revealed 178 reported cases, with a female and thoracic predominance. The majority of patients underwent surgical treatment, achieving a gross total resection in most cases. Similarly, complete symptom resolution was the most common outcome.

Conclusion

Spinal angiolipomas are uncommon spinal tumors. However, they may be treated as any other space-occupying lesion, and surgical resection allows for complete symptom recovery in most patients.

KEYWORDS: SPINAL ANGIOLIPOMA, EPIDURAL SPINAL ANGIOLIPOMA, SPINAL TUMOR, SPINE SURGERY

VOLUME 9 ARTICLE 67 DOI: 10.14444/2067

\section{Introduction}

Spinal angiolipomas (SAL) are uncommon benign tumors composed of mature adipocytes and abnormal blood vessels. They comprise approximately $1.2 \%$ of all spinal axis tumors, and 3\% of all spinal epidural neoplasms. ${ }^{1}$ Given their rarity, these lesions are often overlooked in the differential diagnosis of spaceoccupying lesions within the spinal canal. Though they most commonly have an insidious course, in some cases the initial manifestation is acute paraplegia. $^{2-6}$

Nonetheless, piecemeal excision is achieved in most of these cases, and spinal cord decompression leads Downloaded from http://ijssurgery.com/ by guest on April 25, 2023 to symptom recovery in the majority of patients. ${ }^{1}$

Thus, the purpose of this article is to report on a case of epidural SAL with co-existing lumbar spinal stenosis and to conduct a literature review on the topic.

\section{Case Report}

A 65-year old female with body mass index of $29 \mathrm{~kg} /$ $\mathrm{m}^{2}$ presented with a 6-week history of lower extremity dysesthesia and neurogenic claudication. On physical examination, she had a positive straight leg test at 30 degrees, and reflexes were diminished in both lower extremities; she was neurologically intact.

Magnetic resonance imaging (MRI) revealed significant lumbar spinal stenosis at L4/L5 and L5/S1 (Fig- 
ure 1). Following failure of conservative therapy, she underwent an uneventful L4-S1 bilateral laminoforaminotomy.

The patient experienced significant relief of her symptoms, but three months later she presented to the emergency department with acute onset of severe back pain and history of recent fall due to leg weakness. On physical examination she was hyperreflexic on both lower extremities, and her lower extremity strength was $3 / 5$. A new MRI revealed a space-occupying lesion in the epidural space, causing significant compression on the spinal cord at the levels of T8 - T10 (Figure 2). Additionally, the lesion extended through the intervertebral foramen at T9/ T10. The patient underwent an emergent posterior laminectomy and en bloc resection of a soft, reddish-
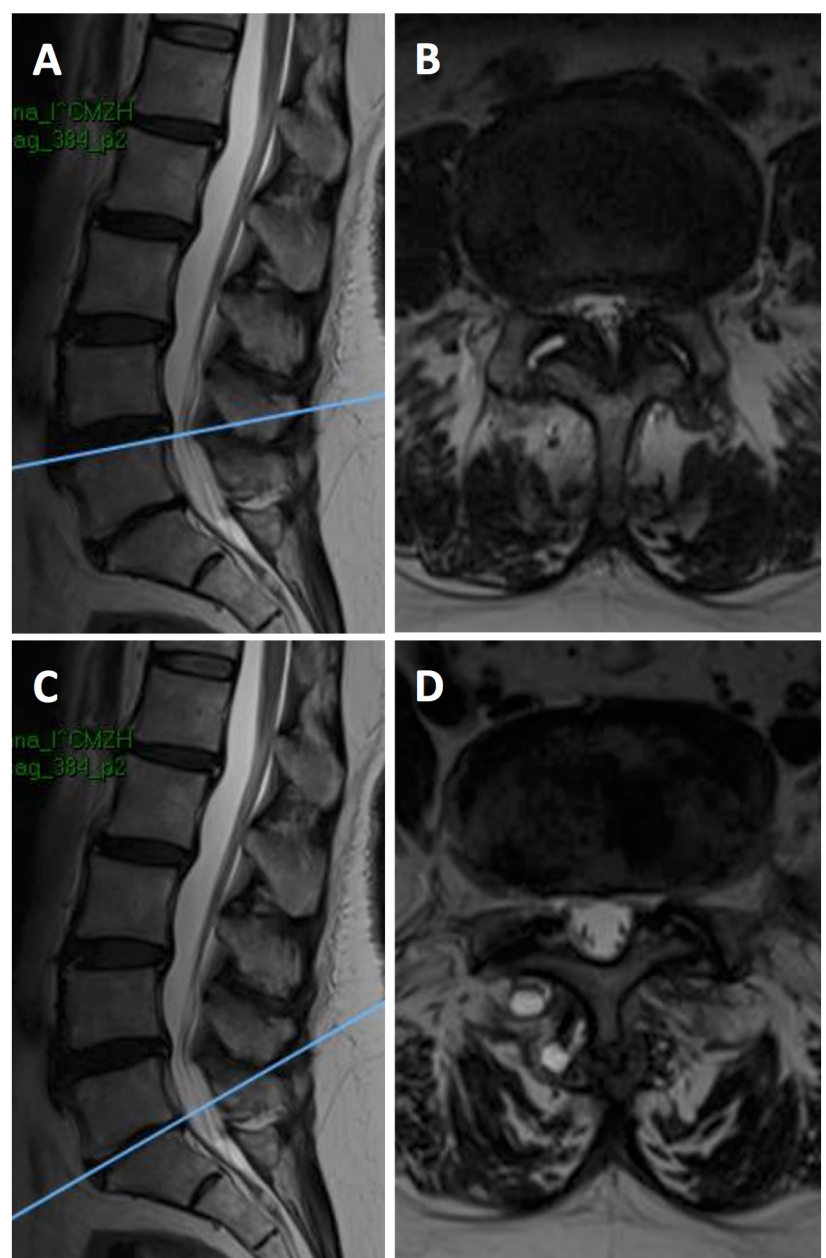

Fig. 1. Magnetic resonance image showing lumbar spinal stenosis. Sagittal (A) and axial (B) T2-weighted images showing lumbar spinal stenosis at L4/L5 secondary to ligamentum flavum hypertrophy and bilateral facet arthropathy. Sagittal (C) and axial (D) T2-weighted images showing foraminal stenosis on the left secondary to facet arthropathy at L5/S1. brown tumor (Figure 3), followed by instrumentation from T7 - T11. Following surgery, histopathological analysis was consistent with angiolipoma (Figure 4). Although the tumor extended through an intervertebral foramen, pathological analysis of surrounding soft tissues revealed no infiltration. The patient underwent an uneventful recovery, and is symptomfree and without evidence of tumor recurrence 6 months after surgery.

\section{Literature Review}

A digital search of the PubMed/Medline databases was performed using the algorithm [("spine" OR "spinal") AND "angiolipoma"] up to June 2015. Article titles and abstracts were then individually screened to identify potential articles of interest, and
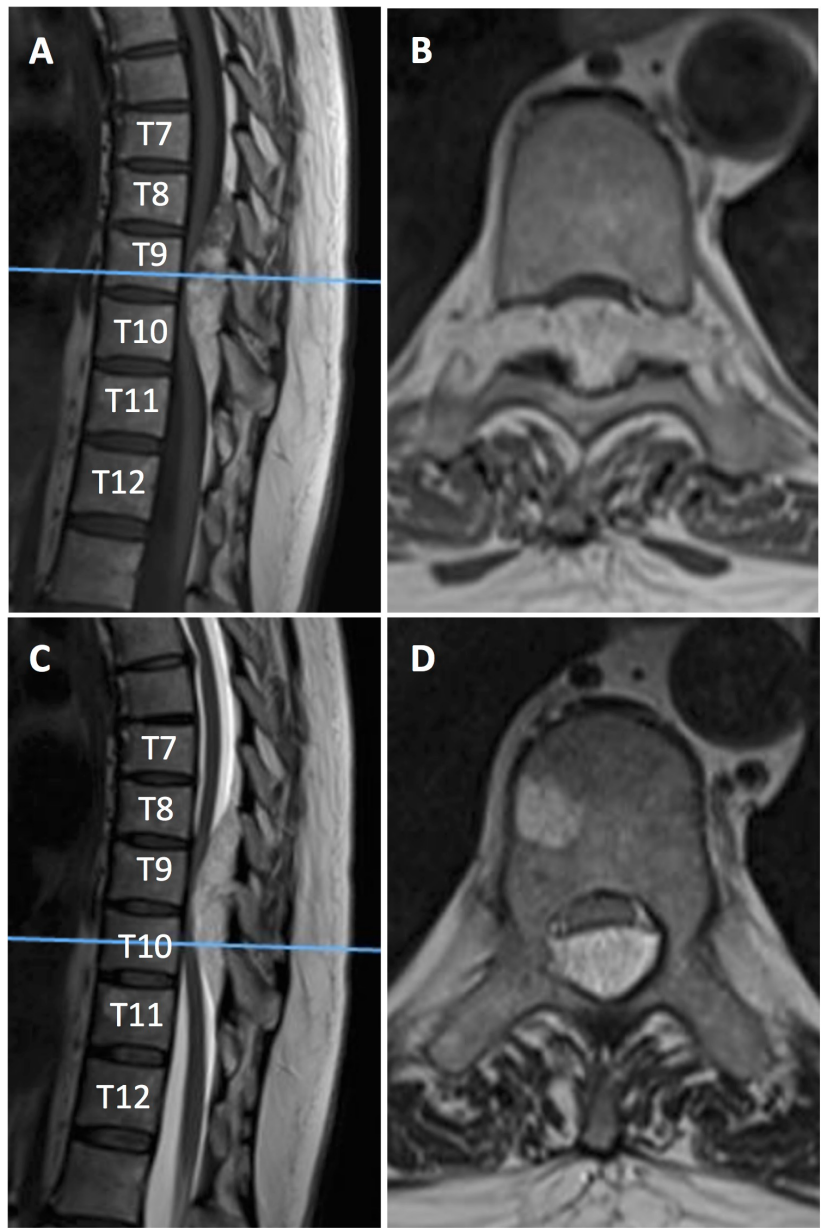

Fig. 2. Magnetic resonance image showing an epidural lesion extending from T8 - T10. Sagittal (A) and axial (B) T1-weighted images showing the dumbbell-shaped hyperintense lesion extending multiple levels and through the foramen at T9/T10. Additionally, the lesion shows hypointense regions, corresponding to the vascular component of the tumor. Sagittal (C) and axial (D) T2-weighted images showing severe spinal stenosis with spinal cord compression. 
selected manuscripts were recorded in a digital database. Article references were also utilized to identify other case reports/case series to reduce publication bias.

A total of 107 articles reporting on 177 patients (108 articles and 178 patients with the present case) with epidural SALs were identified (Table 1) ${ }^{1-107}$ The average age for all patients (including the present case) was $46 \pm 16$ years, with a range of 17 months to 85 years. From the total group, 105 (59\%) patients were female. The most common presenting symptom was paraparesis in $54(30.3 \%)$ patients, followed by thoracic/low back pain in $43(24.2 \%)$ patients. The range of time with symptoms before presentation was from a few minutes (acute onset) to 30 years in one patient. $^{105}$
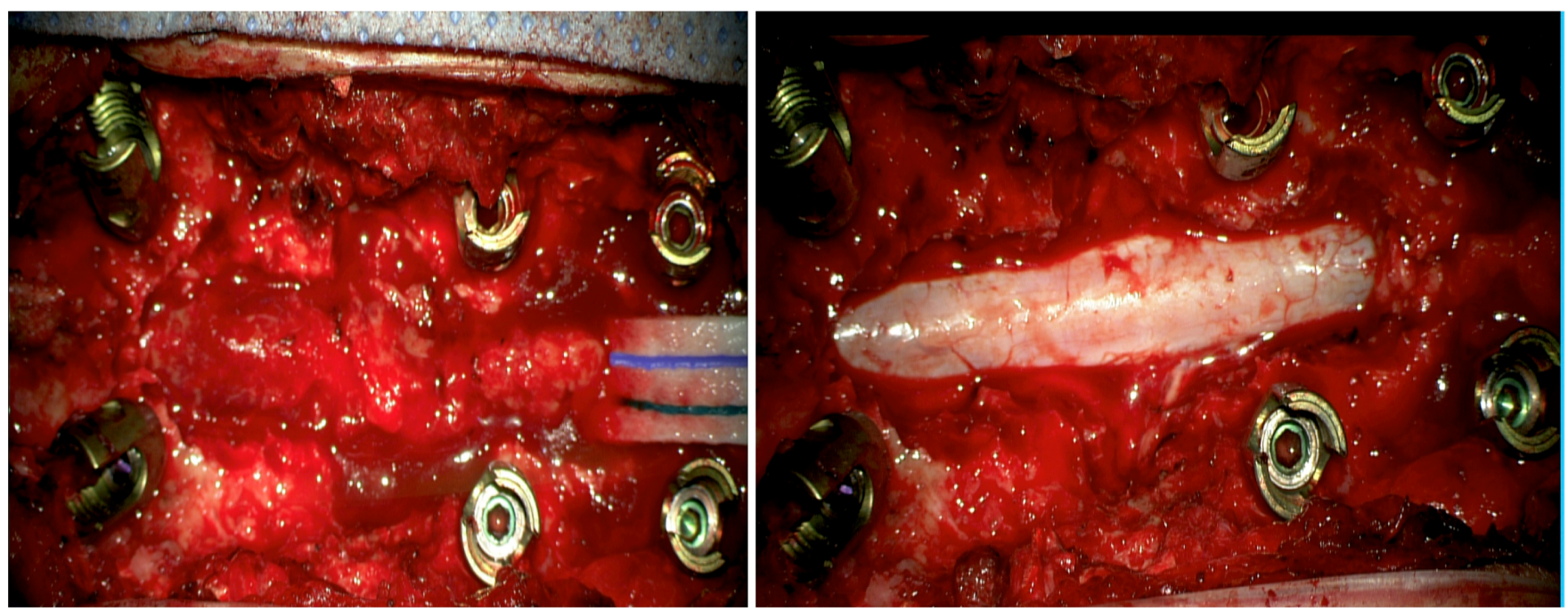

Fig. 3. Intraoperative photographs showing a reddish-brown lesion overlying the thecal sac (Left) which was removed en bloc following laminectomy (Right).

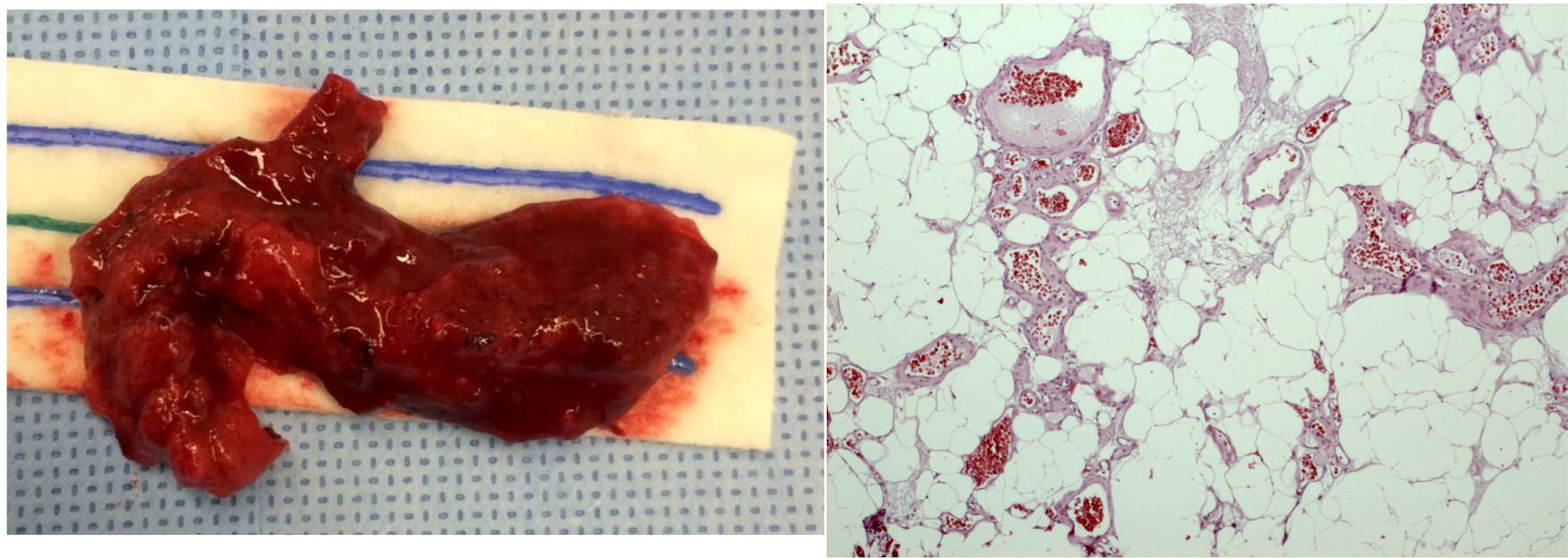

Fig. 4. Tumor specimen consisting of a soft, reddish-brown mass (Left). Histological slide showing mature adipocytes and blood vessels of various sizes, some filled with thrombin (Right) 
Table 1. Review of published cases on epidural spinal angiolipoma.

\begin{tabular}{|c|c|c|c|c|c|c|c|c|}
\hline Author & Year & Age, sex & First symptom & Level & Axial location & Infiltrating & Treatment & Outcome \\
\hline Berenbruch & 1890 & $16 / \mathrm{M}$ & Paraparesis & Thoracic & Not available & $\mathrm{N}$ & Surgery & \\
\hline Liebscher & 1901 & $51 / \mathrm{F}$ & Numbness & T7-T8 & Anterior & $\mathrm{N}$ & No surgery & \\
\hline Frazier and Allen & 1918 & $55 / \mathrm{F}$ & & Thoracic & Unknown & $\mathrm{N}$ & Unknown & Unknown \\
\hline Balado and Morea & 1928 & $20 / \mathrm{F}$ & Paraparesis & Midthoracic & Unknown & $\mathrm{N}$ & No surgery & \\
\hline Kasper and Cowan & 1929 & $6 / M$ & Bleeding to lumbar puncture & $\mathrm{C} 2-\mathrm{T} 1$ and $\mathrm{L} 3-\mathrm{S} 3$ & Unknown & $\mathrm{N}$ & No surgery & \\
\hline Petit-Dutaillis and Christoph & 1931 & $43 / \mathrm{F}$ & Paraplegia & T6-T10 & Unknown & $\mathrm{N}$ & Surgery & Recovery \\
\hline Ehni and Love & 1945 & $30 / \mathrm{F}$ & Paraplegia & T6-T8 & Posterior & $\mathrm{N}$ & Surgery & Recovery \\
\hline Ehni and Love & 1945 & $67 / \mathrm{M}$ & Paraparesis & T8-T9 & Posterior & $\mathrm{N}$ & Surgery & Recovery \\
\hline Bucy and Ritchey & 1947 & $33 / \mathrm{M}$ & Paraparesis & C6-T5 & Posterolateral & $\mathrm{N}$ & Surgery + RT & Improved \\
\hline Taylor et al. & 1951 & $51 / \mathrm{F}$ & Paraparesis & T3-T6 & Posterior & $\mathrm{N}$ & Surgery & Improved \\
\hline Taylor et al. & 1951 & $44 / \mathrm{F}$ & Paraparesis & T10-L1 & Posterior & $\mathrm{Y}$ & Surgery & Improved \\
\hline Moscatelli and Merigliano & 1958 & $51 / \mathrm{M}$ & & T4-T7 & Posterior & $\mathrm{N}$ & Unknown & Unknown \\
\hline Maier & 1962 & $1.5 / \mathrm{F}$ & Paraparesis & $\mathrm{T} 7$ & Anterior & Y & Surgery & Recovery \\
\hline Gonzalez-Crusi et al. & 1966 & $20 / \mathrm{F}$ & LBP & L1-L4 & Anterior & $\mathrm{Y}$ & Surgery + RT & Recovery \\
\hline Gagliardi and Gambacorta & 1968 & $30 / \mathrm{M}$ & Paraparesis & T3-T4 & Posterior & $\mathrm{N}$ & Unknown & Unknown \\
\hline Lo Re and Michelacci & 1969 & $16 / \mathrm{M}$ & Congenital & Cauda & Posterior & $\mathrm{N}$ & Surgery & Recovery \\
\hline Lo Re and Michelacci & 1969 & $35 / \mathrm{F}$ & & Lumbar & Posterior & $\mathrm{N}$ & Surgery & Recovery \\
\hline Warot and Petit & 1969 & $57 / \mathrm{F}$ & Paraparesis & T4-T6 & Posterior & $\mathrm{N}$ & Unknown & Unknown \\
\hline Pearson et al. & 1970 & $17 / \mathrm{F}$ & Back pain & T2-T5 & Anterior & $\mathrm{Y}$ & Surgery & Improved \\
\hline Pearson et al. & 1970 & $22 / \mathrm{M}$ & Back pain & T3-T9 & Anterior & $\mathrm{Y}$ & Surgery & Improved \\
\hline Pearson et al. & 1970 & $44 / \mathrm{F}$ & Numbness & $\mathrm{T} 7-\mathrm{T} 10$ & Posterior & $\mathrm{N}$ & Surgery & Recovery \\
\hline Henry et al. & 1971 & $63 / \mathrm{M}$ & Paraparesis & Cervicothoracic & Posterior & $\mathrm{N}$ & No surgery & Death due to other causes \\
\hline Giuffré & 1971 & $36 / \mathrm{F}$ & & T4-T8 & Unknown & $\mathrm{N}$ & Unknown & Unknown \\
\hline Bender et al. & 1974 & $50 / \mathrm{F}$ & Paraparesis & T6-T8 & Posterior & $\mathrm{N}$ & Surgery & Recovery and late recurrence \\
\hline
\end{tabular}

Downloaded from http://ijssurgery.com/ by guest on April 25, 2023 


\begin{tabular}{|c|c|c|c|c|c|c|c|c|}
\hline Bender et al. & 1974 & $58 / \mathrm{M}$ & Paraparesis & T7-T10 & Posterior & $\mathrm{N}$ & Surgery & Improved \\
\hline Scanarini and Carteri & 1974 & $57 / \mathrm{F}$ & Paraplegia & $\mathrm{T} 7$ & Posterior & $\mathrm{N}$ & Surgery & Recovery \\
\hline Obrador et al. & 1977 & $43 / \mathrm{F}$ & Leg pain & T7-T9 & Posterior & $\mathrm{N}$ & Surgery & Recovery \\
\hline Occhiogrosso \&Vailati & 1977 & $34 / \mathrm{F}$ & Numbness & T6-T8 & Posterior & $\mathrm{N}$ & Surgery & Recovery \\
\hline Cull et al. & 1978 & $50 / \mathrm{F}$ & Paraparesis & T8-T9 & Posterior & $\mathrm{N}$ & Surgery & Recovery \\
\hline Cull et al. & 1978 & $45 / \mathrm{F}$ & Paraparesis & T6-T8 & Posterior & $\mathrm{N}$ & Surgery & Improved \\
\hline Shuangshoti and Hongsagrabhas & 1979 & $45 / \mathrm{M}$ & & $\mathrm{T} 10-\mathrm{T} 11$ & Posterior & $\mathrm{N}$ & Surgery & Recovery \\
\hline Schiffer et al. & 1980 & $48 / \mathrm{F}$ & Numbness & T10-L1 & Anterior & Y & Surgery & Recovery \\
\hline Goyal & 1980 & $67 / \mathrm{F}$ & Paraparesis & & Posterolateral & $\mathrm{N}$ & Surgery & Dead \\
\hline Miki et al. & 1981 & $46 / \mathrm{F}$ & Numbness & T3-T5 & Posterior & $\mathrm{N}$ & Surgery & Recovery \\
\hline Padovani et al. & 1982 & $50 / \mathrm{M}$ & Paraparesis & T4-T6 & Posterolateral & $\mathrm{N}$ & Surgery & Recovery \\
\hline Padovani et al. & 1982 & $52 / \mathrm{M}$ & Paraparesis & $\mathrm{T} 4-\mathrm{T} 6$ & Posterior & $\mathrm{Y}$ & Surgery & Improved \\
\hline Hanakita and Koyama & 1982 & $42 / \mathrm{M}$ & Gait disturbance & T4-T5 & Posterior & $\mathrm{N}$ & Surgery & Recovery \\
\hline Pasquier et al. & 1984 & $48 / \mathrm{F}$ & & T8-T10 & Posterior & $\mathrm{N}$ & Surgery & Improved \\
\hline Butti et al. & 1984 & $44 / \mathrm{M}$ & Paresthesia right arm & $\mathrm{C} 6-\mathrm{C} 7$ & Posterior & $\mathrm{N}$ & Surgery & Recovery \\
\hline Butti et al. & 1984 & $50 / \mathrm{F}$ & Numbness in legs & L4-L5 & Posterior & $\mathrm{N}$ & Surgery & Recovery \\
\hline Bardosi et al. & 1985 & $46 / \mathrm{M}$ & LBP & T11-L2 & Posterior & $\mathrm{N}$ & Surgery & Recovery \\
\hline Von Hanwehr et al. & 1985 & $35 / \mathrm{M}$ & Numbness of right foot & T6 & Anterior & $\mathrm{Y}$ & Surgery & Recovery \\
\hline Haddad et al. & 1986 & $34 / \mathrm{M}$ & Numbness of legs & T5-T6 & Posterior & $\mathrm{Y}$ & Surgery & Improved \\
\hline Haddad et al. & 1986 & $22 / \mathrm{M}$ & Back pain & $\mathrm{T} 7-\mathrm{T} 8$ & Posterior & $\mathrm{N}$ & Surgery & Recovery \\
\hline Griebel et al. & 1986 & $53 / \mathrm{F}$ & Paraparesis & T5-T6 & Posterior & $\mathrm{N}$ & Surgery & Recovery \\
\hline Nishiura et al. & 1986 & $42 / \mathrm{M}$ & Numbness in legs & T3-T6 & Posterior & $\mathrm{N}$ & Surgery & Improved \\
\hline Nishiura et al. & 1986 & $24 / \mathrm{M}$ & LBP & L5-S1 & Anterolateral & $\mathrm{N}$ & Surgery & Recovery \\
\hline Nishiura et al. & 1986 & $45 / \mathrm{M}$ & Numbness in legs & T3-T6 & Posterior & $\mathrm{N}$ & Surgery & Improved \\
\hline Matsushima et al. & 1987 & $41 / \mathrm{F}$ & Paraparesis & T9-T10 & Posterior & $\mathrm{N}$ & Surgery & Recovery \\
\hline Rivkind et al. & 1986 & $\begin{array}{c}52 / \mathrm{M} \\
\text { Downloa }\end{array}$ & $\begin{array}{l}\text { Paraparesis } \\
\text { led from http://iissurgery.com/ by guest on } A\end{array}$ & pril 25,2023 & Anterolateral & $\mathrm{N}$ & Surgery & Recovery \\
\hline
\end{tabular}




\begin{tabular}{|c|c|c|c|c|c|c|c|c|}
\hline Poon et al. & 1988 & $65 / \mathrm{F}$ & Back pain & T8-T9 & Posterior & $\mathrm{N}$ & Surgery & Recovery \\
\hline Parizel & 1989 & $37 / \mathrm{F}$ & & $\mathrm{T} 4-\mathrm{T} 8$ & Unknown & $\mathrm{N}$ & Surgery & \\
\hline Anson et al. & 1990 & $58 / \mathrm{F}$ & Back pain & T2-T6 & Posterolateral & $\mathrm{N}$ & Surgery + RT & Recovery \\
\hline Anson et al. & 1990 & $65 / \mathrm{F}$ & Paraparesis & T1-T8 & Posterior & $\mathrm{N}$ & Surgery & Unchanged \\
\hline Kuroda et al. & 1990 & $73 / \mathrm{F}$ & Numbness in left leg & $\mathrm{T} 4$ & Posterior & $\mathrm{Y}$ & Surgery & Improved \\
\hline Weill et al. & 1991 & $46 / \mathrm{F}$ & Paraparesis & $\mathrm{T} 7-\mathrm{T} 10$ & Posterior & $\mathrm{N}$ & Surgery & Recovery \\
\hline Mascalchi et al. & 1991 & $42 / \mathrm{F}$ & Numbness of left toe & T5-T6 & Posterior & $\mathrm{N}$ & Surgery & Recovery \\
\hline Rubin et al. & 1992 & $58 / \mathrm{M}$ & Paraparesis & T8-T10 & Posterior & $\mathrm{N}$ & Surgery & Improved \\
\hline Stranjalis et al. & 1992 & $68 / \mathrm{F}$ & Paraparesis & T5-T6 & Posterior & $\mathrm{Y}$ & Surgery & Improved \\
\hline Pagni and Canavero & 1992 & $56 / \mathrm{F}$ & LBP & L3 & Anterior & $\mathrm{N}$ & Surgery & Recovery \\
\hline Pagni and Canavero & 1992 & $59 / \mathrm{F}$ & LBP & L4-L5 & Anterior & $\mathrm{N}$ & Surgery & Recovery \\
\hline Mimata & 1992 & $60 / \mathrm{M}$ & & T5-T8 & Unknown & $\mathrm{N}$ & Surgery & Recovery \\
\hline Yamashita et al. & 1993 & $57 / \mathrm{M}$ & Gait disturbance & T3-T9 & Posterior & $\mathrm{N}$ & Surgery & Recovery \\
\hline Shibata & 1993 & $38 / \mathrm{F}$ & Paraparesis & T4-T6 & Unknown & $\mathrm{N}$ & Surgery & Recovery \\
\hline Preul et al. & 1993 & $45 / \mathrm{F}$ & Numbness in legs & T7-T11 & Posterior & $\mathrm{N}$ & Surgery & Recovery \\
\hline Preul et al. & 1993 & $58 / \mathrm{M}$ & Back pain & $\mathrm{T} 3$ & Posterior & $\mathrm{Y}$ & Surgery & Unchanged \\
\hline Michilli et al. & 1993 & $12 / \mathrm{M}$ & LBP & $\mathrm{T} 5-\mathrm{T} 10$ & Posterior & $\mathrm{N}$ & Surgery & Recovery \\
\hline Sakaki et al. & 1993 & $67 / \mathrm{M}$ & Leg pain & T12-L1 & Lateral & $\mathrm{N}$ & Surgery & Recovery \\
\hline Fernandez et al. & 1994 & $14 / \mathrm{F}$ & Paraplegia & $\mathrm{T} 5$ & Posterior & $\mathrm{N}$ & Surgery & Improved \\
\hline Fernandez et al. & 1994 & $28 / \mathrm{M}$ & Paraplegia & C7-T4 & Posterior & $\mathrm{N}$ & Surgery & Unchanged \\
\hline Turanzas et al. & 1994 & $25 / \mathrm{M}$ & Paraparesis & T6-T8 & Anterior & $\mathrm{Y}$ & Surgery & Improved \\
\hline Balbo & 1995 & $41 / \mathrm{M}$ & Paraparesis & T6-T7 & Posterior & $\mathrm{N}$ & Surgery & Recovery \\
\hline Bouramas & 1995 & $27 / \mathrm{F}$ & Numbness in legs & $\mathrm{T} 2-\mathrm{T} 8$ & Posterior & $\mathrm{N}$ & Surgery & Recovery \\
\hline O'Donovan & 1996 & $54 / \mathrm{M}$ & Back pain & T3-T9 & Posterior & $\mathrm{N}$ & Surgery & Recovery \\
\hline Trabulo et al. & 1996 & $26 / \mathrm{F}$ & Back pain & T2-T9 & Posterior & $\mathrm{N}$ & Surgery & Recovery \\
\hline Trabulo et al. & 1996 & $\begin{array}{c}\text { 72/M } \\
\text { Downloa }\end{array}$ & $\begin{array}{l}\text { Paraparesis } \\
\text { ded from http://ijssurgery.com/ by guest on } A\end{array}$ & pril 25,2023 & Posterior & $\mathrm{Y}$ & Surgery & Recovery \\
\hline
\end{tabular}




\begin{tabular}{|c|c|c|c|c|c|c|c|c|}
\hline Provenzale \& McLendon & 1996 & $38 / \mathrm{F}$ & LBP & Lumbar & Posterior & $\mathrm{N}$ & Surgery & Unknown \\
\hline Provenzale \& McLendon & 1996 & $61 / \mathrm{F}$ & Paraparesis & Thoracic (2 tumors) & Posterior & $\mathrm{N}$ & Surgery & Unknown \\
\hline Provenzale \& McLendon & 1996 & $42 / \mathrm{F}$ & Back pain & Thoracic & Posterior & $\mathrm{N}$ & Surgery & Unknown \\
\hline Krishnan et al. & 1996 & $55 / \mathrm{F}$ & Paraparesis & T6-T9 & Posterior & $\mathrm{N}$ & Surgery & Recovery \\
\hline Boockvar et al. & 1997 & $34 / \mathrm{F}$ & Back pain & T3-T9 & Posterior & $\mathrm{N}$ & Surgery & Recovery \\
\hline Shuangshoti \& Lerdlum & 1997 & $21 / \mathrm{M}$ & Paraparesis & $\mathrm{T} 1-\mathrm{T} 2$ & Posterior & $\mathrm{N}$ & Surgery & Recovery \\
\hline Sakaida & 1998 & $72 / \mathrm{M}$ & Numbness in legs & T3-T5 & Anterior & $\mathrm{Y}$ & Surgery & Recovery \\
\hline Labram et al. & 1999 & $40 / \mathrm{F}$ & Back pain & C6-T3 & Posterolateral & $\mathrm{N}$ & Surgery & Recovery \\
\hline Labram et al. & 1999 & $68 / \mathrm{F}$ & Back pain & $\mathrm{T} 5-\mathrm{T} 10$ & Posterior & $\mathrm{N}$ & Surgery & Recovery \\
\hline Labram et al. & 1999 & $17 / \mathrm{M}$ & Back pain & C5-T3 & Circumferential & $\mathrm{N}$ & Surgery & Recovery \\
\hline Turgut & 1999 & $54 / \mathrm{F}$ & Paraparesis & T4-T9 & Posterior & $\mathrm{N}$ & Surgery & Recovery \\
\hline Kujas & 1999 & $67 / \mathrm{F}$ & Paraparesis & T6 & Anterior & $\mathrm{N}$ & Surgery & Worsened \\
\hline Oge et al. & 1999 & $72 / \mathrm{M}$ & Paraparesis & T2-T5 & Posterior & $\mathrm{N}$ & Surgery & Recovery \\
\hline El Abbadi & 1999 & 38 & Paraparesis & T10-T11 & Posterior & $\mathrm{N}$ & Surgery & Recovery \\
\hline Bailey et al. & 2000 & $44 / \mathrm{F}$ & Numbness in legs & T3-T8 & Posterior & $\mathrm{N}$ & Surgery & Recovery \\
\hline Al-Anazi et al. & 2000 & $38 / \mathrm{F}$ & Numbness of both legs & T5-T9 & Posterior & $\mathrm{N}$ & Surgery & Recovery \\
\hline Andaluz et al. & 2000 & $24 / \mathrm{F}$ & Paraparesis & $\mathrm{T} 7-\mathrm{T} 10$ & Posterior & $\mathrm{N}$ & Surgery + embolization & Recovery \\
\hline Andaluz et al. & 2000 & $39 / \mathrm{F}$ & Numbness in legs & T6-T8 & Posterior & $\mathrm{N}$ & Surgery & Recovery \\
\hline Andaluz et al. & 2000 & $59 / \mathrm{F}$ & Paraparesis & T4-T6 & Posterior & $\mathrm{N}$ & Surgery & Recovery \\
\hline Andaluz et al & 2000 & $69 / \mathrm{M}$ & Paraparesis & T3-T4 & Posterior & $\mathrm{N}$ & Surgery & Recovery \\
\hline Akhaddar et al. & 2000 & $47 / \mathrm{F}$ & Numbness in legs & T4-T6 & Posterior & $\mathrm{N}$ & Surgery & Recovery \\
\hline Akhaddar et al. & 2000 & $46 / \mathrm{F}$ & Paraparesis & T7-T9 & Posterior & $\mathrm{N}$ & Surgery & Recovery \\
\hline Akhaddar et al. & 2000 & $38 / \mathrm{F}$ & Numbness in legs & T3-T5 & Posterior & $\mathrm{N}$ & Surgery & Recovery \\
\hline Akhaddar et al. & 2000 & $46 / \mathrm{F}$ & Paraparesis & T3-T4 & Posterior & $\mathrm{N}$ & Surgery & Recovery \\
\hline Akhaddar et al. & 2000 & $12 / \mathrm{F}$ & Paraparesis & T7-T8 & Posterior & $\mathrm{N}$ & Surgery & Improved \\
\hline Akhaddar et al. & 2000 & $\begin{array}{c}\text { 64/F } \\
\text { Downloa }\end{array}$ & $\begin{array}{l}\text { Leg pain } \\
\text { ded from http://iissurgery.com/ by guest on } A\end{array}$ & pril $25,2023^{\mathrm{T} 2-\mathrm{T} 4}$ & Posterolateral & $\mathrm{N}$ & Surgery & Recovery \\
\hline
\end{tabular}




\begin{tabular}{|c|c|c|c|c|c|c|c|c|}
\hline Akhaddar et al. & 2000 & $58 / \mathrm{M}$ & Numbness in legs & T3-T6 & Posterior & $\mathrm{N}$ & Surgery & Recovery \\
\hline Akhaddar et al. & 2000 & $47 / M$ & Back pain & T3-T6 & Posterior & $\mathrm{N}$ & Surgery & Improved \\
\hline Amlashi et al. & 2001 & $36 / \mathrm{F}$ & LBP & T2-T6 & Posterior & $\mathrm{N}$ & Surgery & Improved \\
\hline Fourney et al. & 2001 & $46 / \mathrm{F}$ & Numbness in legs & T6-T8 & Posterior & $\mathrm{N}$ & Surgery & Recovery \\
\hline Garg et al. & 2002 & $12 / \mathrm{F}$ & Paraparesis & T1-T11 & Posterolateral & $\mathrm{N}$ & Surgery & Improved \\
\hline Gelabert et al. & 2002 & $4 / \mathrm{M}$ & Paraparesis & T2-T5 & Posterior & $\mathrm{N}$ & Surgery & Recovery \\
\hline Pinto et al. & 2002 & $85 / \mathrm{M}$ & Leg pain & L1-L2 & Posterior & $\mathrm{N}$ & Surgery & Improved \\
\hline Samdani et al. & 2004 & $49 / \mathrm{F}$ & LBP & T6-T8 & Posterior & $\mathrm{N}$ & Surgery & Recovery \\
\hline Aversa do Souto et al. & 2003 & $46 / \mathrm{F}$ & LBP & L4-L5 & Anterior & $\mathrm{N}$ & Surgery & Recovery \\
\hline Rocchi et al. & 2004 & $60 / \mathrm{M}$ & Leg pain & L3-L4 & Anterior & $\mathrm{N}$ & Surgery & Recovery \\
\hline Rocchi et al. & 2004 & $54 / \mathrm{F}$ & Leg pain & L3 & Anterior & $\mathrm{N}$ & Surgery & Recovery \\
\hline Rabin et al. & 2004 & $47 / \mathrm{M}$ & Numbness in legs & T9 & Anterior & $\mathrm{Y}$ & Embolization + Surgery & Recovery \\
\hline Petrella et al. & 2005 & $16 / \mathrm{M}$ & LBP & T4-T8 & Posterior & $\mathrm{N}$ & Surgery & Recovery \\
\hline Cubillos et al. & 2005 & $40 / \mathrm{F}$ & Numbness in legs & T4-T7 & Posterior & $\mathrm{N}$ & Surgery & Recovery \\
\hline Konya et al. & 2006 & $60 / \mathrm{F}$ & LBP & L5 & Posterolateral & $\mathrm{N}$ & Surgery & Recovery \\
\hline Dogan et al. & 2006 & $50 / \mathrm{F}$ & Leg pain & L4-L5 & Posterior & $\mathrm{N}$ & Surgery & Recovery \\
\hline Dogan et al. & 2006 & $36 / \mathrm{M}$ & LBP & L2 & Posterior & $\mathrm{N}$ & Surgery & Recovery \\
\hline Raghavendra et al & 2007 & $14 / \mathrm{F}$ & Subarachnoid hemorrhage and acute back pain & T1-T9 & Posterior & $\mathrm{N}$ & No surgery & Unchanged \\
\hline Guzey et al. & 2007 & $41 / \mathrm{F}$ & LBP and leg pain & L2-L3 & Posterolateral & $\mathrm{Y}$ & Surgery & Recovery \\
\hline Akhaddar et al. & 2008 & $47 / \mathrm{M}$ & Paraparesis & $\mathrm{T} 2-\mathrm{T} 3$ & Posterior & $\mathrm{N}$ & Surgery & Recovery \\
\hline Nanassis et al. & 2008 & $47 / \mathrm{F}$ & LBP and leg pain & L2-L3 & Posterior & $\mathrm{N}$ & Surgery & Recovery \\
\hline Farooq et al. & 2008 & $57 / \mathrm{F}$ & LBP and numbness in legs & $\mathrm{T} 5-\mathrm{T} 8$ & Posterior & $\mathrm{N}$ & Surgery & Recovery \\
\hline Yen et al. & 2008 & $71 / \mathrm{M}$ & Back pain and progressive paraparesis & T5-T6 & Posterior & $\mathrm{Y}$ & Surgery & Recovery \\
\hline Hungs et al. & 2008 & $52 / \mathrm{F}$ & Back pain and leg pain & $\mathrm{T} 2-\mathrm{T} 5$ & Posterior & $\mathrm{N}$ & Surgery & Recovery \\
\hline Park et al. & 2008 & $74 / \mathrm{M}$ & LBP & L5-S1 & Lateral & $\mathrm{N}$ & Surgery & Recovery \\
\hline Gelabert-Gonzalez et al. & 2009 & $\begin{array}{c}16 / \mathrm{M} \\
\text { Downloa }\end{array}$ & $\begin{array}{c}\text { LBP } \\
\text { ded from http://iissurgery com/ by guest on } A\end{array}$ & April $25,2023^{\text {L5-S1 }}$ & Posterior & $\mathrm{N}$ & Surgery & Recovery \\
\hline
\end{tabular}




\begin{tabular}{|c|c|c|c|c|c|c|c|c|}
\hline Gelabert-Gonzalez et al. & 2009 & $45 / \mathrm{F}$ & Paraparesis and numbness & L5-S1 & Posterior & $\mathrm{N}$ & Surgery & Improved \\
\hline Dufrenot et al. & 2010 & $44 / \mathrm{F}$ & & & & & Surgery & Recovery \\
\hline Sankaran et al. & 2010 & $77 / \mathrm{M}$ & Post-traumatic bleeding and acute paraparesis & T8-T10 & Posterior & $\mathrm{N}$ & Surgery & Recovery \\
\hline Haji et al. & 2011 & $65 / \mathrm{F}$ & Numbness in legs & T5-T7 & Posterior & $\mathrm{N}$ & Surgery & Improved \\
\hline Diyora et al. & 2011 & 20/M & Back pain and paraparesis & T5-T8 & Posterior & $\mathrm{N}$ & Surgery & Recovery \\
\hline Tsutsumi et al. & 2011 & $31 / \mathrm{F}$ & Paraplegia & T3-T4 & Posterolateral & $\mathrm{N}$ & Surgery & Recovery \\
\hline Chotai et al. & 2011 & $68 / \mathrm{M}$ & Back pain and numbness in legs & T9-T11 & Posterior & $\mathrm{N}$ & Surgery & Recovery \\
\hline Han et al. & 2012 & $58 / \mathrm{M}$ & Leg dysesthesia and paraparesis & T4-T5 & Posterolateral & $\mathrm{Y}$ & Surgery & Recovery \\
\hline Ghanta et al. & 2012 & $56 / \mathrm{M}$ & Leg numbness and gait disturbance & T4-T5 & Posterior & $\mathrm{N}$ & Surgery & Improved \\
\hline Fujiwara et al. & 2013 & $64 / \mathrm{F}$ & Leg dysesthesia & T5-T8 & Posterior & $\mathrm{N}$ & Surgery & Recovery \\
\hline Fujiwara et al. & 2013 & $65 / \mathrm{M}$ & Leg dysesthesia and gait disturbance & T5-T7 & Posterior & $\mathrm{N}$ & Surgery & Recovery \\
\hline Reyes et al. & 2013 & $68 / \mathrm{M}$ & LBP & T10-L1 & Posterior & $\mathrm{N}$ & Surgery & Recovery \\
\hline Si et al. & 2014 & $50 / \mathrm{M}$ & NR & L3-L4 & Posterior & $\mathrm{N}$ & Surgery & \\
\hline Si et al. & 2014 & $53 / \mathrm{M}$ & NR & T4-T7 & Posterior & $\mathrm{N}$ & Surgery & Improved \\
\hline Si et al. & 2014 & $58 / \mathrm{M}$ & NR & T9-T10 & Posterior & $\mathrm{N}$ & Surgery & Recovery \\
\hline Si et al. & 2014 & $41 / \mathrm{F}$ & NR & T5-T6 & Posterior & $\mathrm{N}$ & Surgery & \\
\hline Si et al. & 2014 & $19 / \mathrm{F}$ & NR & $\mathrm{C} 3-\mathrm{C} 6$ & Posterior & $\mathrm{N}$ & Surgery & \\
\hline Si et al. & 2014 & $26 / \mathrm{F}$ & NR & $\mathrm{C} 4-\mathrm{C} 6$ & Posterolateral & $\mathrm{N}$ & Surgery & \\
\hline Si et al. & 2014 & $63 / \mathrm{F}$ & NR & $\mathrm{T} 7-\mathrm{T} 10$ & Posterior & $\mathrm{N}$ & Surgery & Improved \\
\hline Si et al. & 2014 & $62 / \mathrm{F}$ & NR & T8-T10 & Posterior & $\mathrm{N}$ & Surgery & Recovery \\
\hline Si et al. & 2014 & $74 / \mathrm{F}$ & NR & L4-L5 & Lateral & $\mathrm{N}$ & Surgery & Recovery \\
\hline Si et al. & 2014 & $55 / \mathrm{M}$ & NR & T3-T5 & Lateral & $\mathrm{N}$ & Surgery & Recovery \\
\hline Si et al. & 2014 & $62 / \mathrm{M}$ & NR & L4-L5 & Posterior & $\mathrm{Y}$ & Surgery & Recovery \\
\hline Si et al. & 2014 & $61 / \mathrm{M}$ & NR & T11-L3 & Posterior & $\mathrm{Y}$ & Surgery & \\
\hline Si et al. & 2014 & $43 / F$ & NR & T7-T9 & Posterolateral & $\mathrm{N}$ & Surgery & Improved \\
\hline Si et al. & 2014 & $\begin{array}{c}\text { 57/M } \\
\text { Downloa }\end{array}$ & led from http://iissurgery.com/ by guest on $A$ & April $25,2023^{\text {T5-T8 }}$ & Posterior & $\mathrm{N}$ & Surgery & \\
\hline
\end{tabular}




\begin{tabular}{|c|c|c|c|c|c|c|c|c|}
\hline Si et al. & 2014 & $69 / \mathrm{F}$ & NR & T4-T6 & Posterolateral & $\mathrm{N}$ & Surgery & Unchanged \\
\hline Si et al. & 2014 & $62 / \mathrm{M}$ & NR & T4-T6 & Posterior & $\mathrm{N}$ & Surgery & Improved \\
\hline Si et al. & 2014 & $47 / \mathrm{F}$ & NR & $\mathrm{T} 2-\mathrm{T} 3$ & Posterior & $\mathrm{Y}$ & Surgery & Improved \\
\hline Si et al. & 2014 & $50 / \mathrm{F}$ & NR & $\mathrm{T} 2-\mathrm{T} 4$ & Posterolateral & $\mathrm{N}$ & Surgery & \\
\hline Si et al. & 2014 & $37 / \mathrm{M}$ & NR & $\mathrm{T} 4-\mathrm{T} 7$ & Posterior & $\mathrm{N}$ & Surgery & \\
\hline Si et al. & 2014 & $51 / \mathrm{F}$ & NR & T4-T5 & Posterior & $\mathrm{N}$ & Surgery & \\
\hline Si et al. & 2014 & $59 / \mathrm{F}$ & NR & T8-T11 & & & Surgery & \\
\hline Ramdasi et al. & 2014 & $58 / \mathrm{M}$ & Paraplegia & $\mathrm{C} 7-\mathrm{T} 1$ & & & Surgery & Recovery \\
\hline Wang et al. & 2014 & $47 / \mathrm{F}$ & Paraparesis & T3-T10 & Posterior & $\mathrm{N}$ & Surgery & Improved \\
\hline Wang et al. & 2014 & $36 / \mathrm{M}$ & Leg pain & L3-S2 & Posterior & $\mathrm{N}$ & Surgery & Recovery \\
\hline Wang et al. & 2014 & $46 / \mathrm{F}$ & Back pain & $\mathrm{T} 2-\mathrm{T} 4$ & Lateral & $\mathrm{Y}$ & Surgery & Recovery \\
\hline Wang et al. & 2014 & $50 / \mathrm{M}$ & Back pain & $\mathrm{T} 1-\mathrm{T} 3$ & Posterior & $\mathrm{N}$ & Surgery & Recovery \\
\hline Wang et al. & 2014 & $47 / \mathrm{F}$ & Numbness in legs & L2-L4 & Posterior & $\mathrm{N}$ & Surgery & Improved \\
\hline Wang et al. & 2014 & $46 / \mathrm{M}$ & Paraparesis & T11-T12 & Posterior & $\mathrm{N}$ & Surgery & Recovery \\
\hline Wang et al. & 2014 & $54 / \mathrm{M}$ & Leg pain & L3-L4 & Posterolateral & $\mathrm{Y}$ & Surgery & Improved \\
\hline Wang et al. & 2014 & $44 / \mathrm{F}$ & Numbness in legs & T8-T11 & Posterior & $\mathrm{N}$ & Surgery & Recovery \\
\hline Wang et al. & 2014 & $55 / \mathrm{F}$ & Leg pain & L5-S1 & Posterior & $\mathrm{N}$ & Surgery & Improved \\
\hline Wang et al. & 2014 & $47 / M$ & LBP & L2-L3 & Posterior & $\mathrm{N}$ & Surgery & Improved \\
\hline Wang et al. & 2014 & $49 / \mathrm{F}$ & LBP & L2-L3 & Posterior & $\mathrm{N}$ & Surgery & Recovery \\
\hline Wang et al. & 2014 & $55 / \mathrm{F}$ & Numbness in legs & T6-T8 & Posterior & $\mathrm{N}$ & Surgery & Improved \\
\hline da Costa et al. & 2014 & $43 / \mathrm{M}$ & Paraplegia & T9-T10 & Posterior & $\mathrm{N}$ & Surgery & Improved \\
\hline Nakao et al. & 2014 & $32 / \mathrm{F}$ & Leg numbness and paraparesis & T1-T6 & Posterior & $\mathrm{N}$ & Surgery & Recovery \\
\hline Nadi et al. & 2015 & $50 / \mathrm{F}$ & Back pain and paraparesis & T6-T9 & Posterolateral & $\mathrm{Y}$ & Surgery & Improved \\
\hline Present case & 2015 & $65 / \mathrm{F}$ & LBP and paraparesis & $\mathrm{T} 8-\mathrm{T} 10$ & Posterolateral & $\mathrm{N}$ & Surgery & Recovery \\
\hline
\end{tabular}

M: male; F: female; LBP: low back pain; NR: not reported; N: no; Y: yes; RT: radiotherapy. 
The majority of tumors were located in the thoracic spine (131 patients; 73.6\%), followed by the lumbosacral spine in 30 patients (16.9\%), cervicothoracic and thoraco-lumbar in 6 patients each $(6.8 \%$ combined) and the cervical spine in only 4 cases (2.3\%). ${ }^{10,35,105}$ Tumors were most commonly located posteriorly in the axial plane. Based on the classification by Lin \& Lin, ${ }^{108}$ only 25 (14\%) tumors were found to be infiltrating in nature. The majority of patients underwent surgery, and experienced complete symptom recovery. Recurrences were uncommon, and have been reported in only two occasions $(1.1 \%){ }^{23,69}$

\section{Discussion}

Angiolipomas are histologically benign tumors that most commonly occur in subcutaneous tissue in the forearms, trunk, neck, and proximal upper extremities. ${ }^{109}$ Occurrence within the spinal canal is uncommon, but when it occurs it is most commonly in the epidural space. ${ }^{1}$ Histologically, these lesions consist of "mature adipocytes and branching capillary-sized vessels, which usually contain fibrin thrombi."109 Some studies have also performed immunohistochemical staining, showing positivity for CD31, Factor XIIIa, and Factor VIII. ${ }^{84}$ Some believe SALs originate from pluripotential mesenchymal stem cells, ${ }^{12}$ but others argue they most likely represent a "congenital malformation or a benign hamartoma" 1 or that they arise from primitive mesenchyme. ${ }^{70}$

SALs resemble other space-occupying lesions in terms of symptoms, and most patients present with a history of thoracic/low back pain and progressive lower extremity weakness. However, paraparesis or paraplegia may also occur acutely, particularly in the setting of tumor bleeding or thrombosis. ${ }^{2-6,65,96}$ A presumed diagnosis can be usually made by MRI, although the ultimate diagnosis is made with biopsy. Lesions typically appear hyperintense on noncontrast T1-weighted images due to their fatty content. ${ }^{110}$ Additionally, there may be hypointense regions on T1-weighted images, which represent the vascular component of the tumor. Thus, these regions will be enhancing after contrast administration. ${ }^{110}$ Computed tomography scans are less frequently utilized, but may show tumor calcification, ${ }^{63,73}$ vertebral body trab ecula tion, ${ }^{14,72}$ and/or ver- tebral bo dy erosion. ${ }^{73}$ Though most SALs are found in the epidural space (as mentioned previously), there have been several reports of intradural (including intramedullary) locations. ${ }^{111-116}$

Historically, SALs have been classified as noninfiltrating and infiltrating, ${ }^{108}$ with the latter referring to tumoral infiltration into the vertebral body and/or adjacent soft tissues. In the present review, only $13.4 \%$ of tumors were found to be infiltrating in nature. More recently, $\mathrm{Si}$ et al. proposed another classification system based on tumor characteristics observed on MRI. This group of authors described three tumor types: Type IA (intraspinal tumor without lipomatosis), Type IB (intraspinal tumor with lipomatosis above and below the tumor) and Type II (dumbbell-shaped tumor). ${ }^{105}$ The rationale behind this classification was that Type IB tumors occur more frequently in obese patients and are more challenging from a surgical standpoint owing to the extensive associated epidural lipomatosis. ${ }^{105}$ In contrast, Type IA tumors are more easily resectable, and Type II represent the most complex type of tumor that may warrant additional internal fixation. ${ }^{105}$

Treatment of SALs is surgical, which was performed in 168 out of 178 patients (94.4\%) in the present review. Additionally, adjuvant radiation therapy was used in three cases (1.7\%) due to suspicion of malignancy. ${ }^{13,17,46}$ Gross total resection was achieved in most cases, but in a subset of patients (particularly those with infiltrating tumors) only subtotal resection was achieved. ${ }^{69}$ Of seven reported cases of intradural/intramedullary SALs, complete excision was only achieved in one, ${ }^{113}$ and most patients only experienced partial improvement in symptoms. When examining global outcomes, we found that the majority of patients experience complete symptom resolution, even for patients presenting acutely with paraplegia.

The case described in this article represented a distinctive challenge given the initial presentation with neurogenic claudication and concomitant finding of lumbar spinal stenosis. Additionally, the acute presentation of paraparesis suggested tumoral thrombosis or bleeding, as it has been reported in other cases. ${ }^{65,96}$ Fortunately, following decompression and piecemeal excision, the patient was able to regain full 
strength postoperatively.

While screening the entire spinal column (with MRI) in cases of suspected lumbar spinal stenosis is not common practice, there may be several indications for cervico-thoracic spine screening. Paraparesis without other signs/symptoms of cauda equina syndrome is rarely due to lumbar stenosis, and may be due to myelopathy and/or other neurological diseases. Patients who present with signs/symptoms of stenosis but with other concomitant findings such as hyperreflexia or spastic gait may require cervicothoracic spine screening to rule-out spinal cord compression or disease. Lastly, though occurrence of multiple SALs in the same patient has been reported, it is unusual (only 2 cases in the present literature review). ${ }^{10,63}$ Each case should be individualized, and a thorough history and physical exam should dictate whether a patient requires cervical, thoracic, and/or lumbar spine imaging.

\section{Conclusion}

SALs are infrequent entities that mimic other spaceoccupying lesions within the spinal canal. Though not common, they should be included in the differential diagnoses of epidural tumors, given the excellent outcome that may be achieved in most patients.

\section{References}

1. Gelabert-Gonzalez M, Garcia-Allut A. Spinal extradural angiolipoma: report of two cases and review of the literature. Eur Spine J. Mar

2009;18(3):324-335.

2. Akhaddar A, Albouzidi A, Elmostarchid B, Gazzaz M, Boucetta M. Sudden onset of paraplegia caused by hemorrhagic spinal epidural angiolipoma. A case report. Eur Spine J. Sep 2008;17 Suppl 2:S296-298.

3. Raghavendra S, Krishnamoorthy T, Ashalatha R, Kesavadas C. Spinal angiolipoma with acute subarachnoid hemorrhage. J Clin Neurosci. Oct 2007;14(10):992-994.

4. Ramdasi RV, Avinasha KM, Mahore A, Kawale J. Spinal angiolipoma manifesting with apoplexy. $B M J$ Case Rep. 2014;2014.
5. Tsutsumi S, Nonaka Y, Abe Y, Yasumoto Y, Ito M. Spinal angiolipoma in a pregnant woman presenting with acute epidural hemorrhage. J Clin Neurosci. Jun 2011;18(6):849-851.

6. Fernández JJ, Abad RM, Ribas T, et al. Spinal angiolipoma causing acute paraplegia. Report of two cases. Neurocirugia. 1994(5):242-245.

7. Barenbruch K. En fall von multiplen angiolipomen kombiniert mit einem angiom des ruckenmarks. Tubingen. 1890.

8. Liebscher C. Angiolipom des Wirbelkanals mit Kompression des Ruckenmarks. Pag Med Wochenschr. 1901(26):189-191.

9. Frazier $\mathrm{CH}$, Allen AR. Surgery of the spine and spinal cord. Appleton, New York1918.

10. Kasper JA, Cowan A. Extradural lipoma of the spinal canal. Arch Pathol (Chic). 1929(8):800-802.

11. Petit-Dutaillis D, Christoph J. Spinal cord copression due to a giant extradural angiolipoma: Surgical removal. Rev Neurol (Paris). 1931(2):824-827.

12. Ehni G, Love JG. Intraspinal lipomas: report of cases, review of literature, and clinical and pathological study. Arch Neurol Psychiatry. 1945;53:1-28.

13. Bucy PC, Ritchey H. Klippel-Feil's syndrome associated with compression of the spinal cord by an extradural hemangiolipoma. J Neurosurg. Sep 1947;4(5):476-481.

14. Taylor J, Harries BJ, Schurr PH. Extrathecal haemangiolipomas of the spinal canal. Br J Surg. Jul 1951;39(153):1-7.

15. Moscatelli G, Merigliano D. [Histogenetic and nosographic aspects of extradural lipoma: a case report]. Riv Neurol. Nov-Dec 1958;28(6):667-676.

16. Maier HC. Extradural and intrathoracic lipoma causing spinal cord compression. Successful treatment by surgical excision. JAMA. Aug 18 1962;181:610-612.

17. Gonzalez-Crussi F, Enneking WF, Arean VM. Infiltrating angiolipoma. J Bone Joint Surg Am. Sep 1966;48(6):1111-1124.

18. Gagliardi FM, Gambacorta D. Extradural thoracic angiolipoma. Gazz Int Med Chir. 1968(73):5602. 19. Lo Re F, Michelacci M. [Clinical and surgical findings on various lumbo-sacral abnormalities associated with angiolipoma]. Arch Putti Chir Organi Mov. 1969;24:70-85.

20. Warot P, Petit H, Christiaens JL, Caron JC, 
Delobelle-Deroide A. [Extra-dural dorsal angiolipoma: unusual case of medullary compression]. Lille Med. Mar 1969;14(3):311-315.

21. Pearson J, Stellar S, Feigin I. Angiomyolipoma: long-term cure following radical approach to malignant-appearing benign intraspinal tumor. Report of three cases. $J$ Neurosurg. Oct 1970;33(4):466-470.

22. Henry P, Vital C, Vallat JM. Anatomoclinical study of an extradural angiolipoma associated with intramedullary glioblastoma. Rev Neurol (Paris). 1971(124):385-391.

23. Bender JL, Van Landingham JH, Manno NJ. Epidural lipoma producing spinal cord compression. Report of two cases. J Neurosurg. Jul 1974;41(1):100-103.

24. Scanarini $M$, Carteri A. Intrarachidian lipomas. Observations on 4 cases and pathogenetic considerations. J Neurosurg Sci. Apr-Jun 1974;18(2):136-141. 25. Giuffré R. Spinal lipomas. In: Vinken AJ, Bryn GN, eds. Handbook of clinical neurology. Vol 20. North-Holland, Amsterdam1976:389-414. 26. Obrador S, Villarejo F, Deblas A. [Extradural angiolipoma: uncommon cause of spinal pressure]. Rev Clin Esp. Sep 30 1977;146(6):395-396.

27. Occhiogrosso M, Vailati G. Medullar compression due to epidural angiolipoma: Report of a case. Acta Neurol (Napoli). 1977(32):850-853.

28. Cull DJ, Erdohazi M, Symon L. Extradural haemangiolipoma in the spinal canal. Two cases presenting during pregnancy. Acta Neurochir (Wien). 1978;45(1-2):187-193.

29. Shuangshoti $S$, Hongsaprabhas C. Intraspinal epidural angiolipoma. J Med Assoc Thai. Aug 1979;62(8):457-460.

30. Goyal RN. Epidural lipoma causing compression of the spinal cord. Surg Neurol. Jul 1980;14(1):77-79.

31. Schiffer J, Gilboa Y, Ariazoroff A. Epidural angiolipoma producing compression of the cauda equina. Neurochirurgia (Stuttg). May 1980;23(3):117-120. 32. Miki T, Oka M, Shima M, Hirofuji E, Tanaka S. Spinal angiolipoma. A case report. Acta Neurochir (Wien). 1981;58(1-2):115-119.

33. Hanakita J, Koyama T. [Spinal epidural hemangiolipoma - a case report (author's transl)]. No Shinkei Geka. Mar 1982;10(3):313-316.
34. Padovani R, Tognetti F, Speranza S, Pozzati E. Spinal extrathecal hemangiolipomas: report of two cases and review of the literature. Neurosurgery. Nov 1982;11(5):674-677.

35. Butti G, Gaetani P, Scelsi M, Pezzotta S. Extradural spinal lipomas. Report of two cases and review of the literature. Neurochirurgia (Stuttg). Jan 1984;27(1):28-30.

36. Pasquier B, Vasdev A, Gasnier F, et al. [Epidural angiolipoma: a rare and curable cause of spinal cord compression]. Ann Pathol. Dec 1984;4(5):365-369.

37. Bardosi A, Schaake T, Friede RL, Roessmann U. Extradural spinal angiolipoma with secretory activity. An ultrastructural, clinico-pathological study. Virchows Arch A Pathol Anat Histopathol. 1985;406(2):253-259.

38. von Hanwehr R, Apuzzo ML, Ahmadi J, Chandrasoma P. Thoracic spinal angiomyolipoma: case report and literature review. Neurosurgery. Mar 1985;16(3):406-411.

39. Griebel RW, Khan M, Rozdilsky B. Spinal extradural angiolipoma. A case report and literature review. Spine (Phila Pa 1976). Jan-Feb 1986;11(1):47-48. 40. Haddad FS, Abla A, Allam CK. Extradural spinal angiolipoma. Surg Neurol. Nov 1986;26(5):473-486.

41. Nishiura I, Kubo Y, Koyama T. Spinal haemangiolipoma. Three case reports. Neurochirurgia (Stuttg). Mar 1986;29(2):63-66.

42. Rivkind A, Margulies JY, Lebensart P, Sherman $\mathrm{Y}$, Robin GC. Anterior approach for removal of spinal angiolipoma. A case report. Spine (Phila Pa 1976). Jul-Aug 1986;11(6):623-625.

43. Matsushima K, Shinohara Y, Yamamoto M, Tanigaki T, Ikeda A, Satoh O. Spinal extradural angiolipoma: MR and CT diagnosis. J Comput Assist Tomogr. Nov-Dec 1987;11(6):1104-1106.

44. Poon TP, Behbahani M, Matoso IM, Katz MA, Pearl M. Epidural angiolipoma with spinal cord compression. J Natl Med Assoc. Mar 1988;80(3):347-349. 45. Parizel PM, Baleriaux D, Rodesch G, et al. GdDTPA-enhanced MR imaging of spinal tumors. $A J R$ Am J Roentgenol. May 1989;152(5):1087-1096.

46. Anson JA, Cybulski GR, Reyes M. Spinal extradural angiolipoma: a report of two cases and review of the literature. Surg Neurol. Sep 1990;34(3):173-178. 
47. Kuroda $\mathrm{S}$, Abe $\mathrm{H}$, Akino $\mathrm{M}$, Iwasaki $\mathrm{Y}, \mathrm{Na}$ gashima $\mathrm{K}$. Infiltrating spinal angiolipoma causing myelopathy: case report. Neurosurgery. Aug 1990;27(2):315-318.

48. Mascalchi M, Arnetoli G, Dal Pozzo G, Canavero S, Pagni CA. Spinal epidural angiolipoma: MR findings. AJNR Am J Neuroradiol. Jul-Aug 1991;12(4):744-745.

49. Weill A, del Carpio-O'Donovan R, Tampieri D, Melanson D, Ethier R. Spinal angiolipomas: CT and MR aspects. J Comput Assist Tomogr. Jan-Feb 1991;15(1):83-85.

50. Mimata C, Wada H, Sano Y, Saitou Y, Kitamura I, Ushio Y. [Spinal extradural angiolipoma: a case report]. No Shinkei Geka. Oct 1992;20(10):1085-1089. 51. Pagni CA, Canavero S. Spinal epidural angiolipoma: rare or unreported? Neurosurgery. Oct 1992;31(4):758-764; discussion 764.

52. Rubin G, Gornish M, Sandbank J, Shevach I, Rappaport ZH. Spinal extradural angiolipoma. Case report and review of the literature. Spine (Phila Pa 1976). Jun 1992;17(6):719-724.

53. Stranjalis G, Jamjoom A, Torrens MJ. MRI in the diagnosis of spinal extradural angiolipoma. $\mathrm{Br} J$ Neurosurg. 1992;6(5):481-483.

54. Michilli R, Tzonos P, Iglesias-Rozas JR. Spinal extradural angiolipoma: case report and literature review. Neurochirurgia (Stuttg). Mar 1993;36(2):63-65. 55. Sakaki T, Fujita T, Yoshimine T, Taneda M, Hayakawa T. [Spinal extradural angiolipoma: a case report]. No Shinkei Geka. Oct 1993;21(10):959-962. 56. Shibata Y, Sugimoto K, Matsuki T, Nose T. Thoracic epidural angiolipoma--case report. Neurol Med Chir (Tokyo). May 1993;33(5):316-319.

57. Yamashita K, Fuji T, Nakai T, Hamada H, Kotoh K. Extradural spinal angiolipoma: report of a case studied with MRI. Surg Neurol. Jan 1993;39(1):49-52. 58. Turanzas FS, Domínguez J, Eyerbe P, Dhimes P. Infiltrating spinal angiolipoma: Case repot. Neurocirugia. 1994(5):246-249.

59. Balbo RJ, Araujo JF, Melro CA, Iafigliola MG, Valvassore FR. [Thoracic epidural angiolipoma. Report of a case]. Arq Neuropsiquiatr. Sep 1995;53(3-B):659-661.

60. Bouramas D, Korres DS, Roussos L, Mantzilas $\mathrm{T}$, Anagnostopoulos D. Spinal extradural angiolipoma. J Spinal Disord. Aug 1995;8(4):324-327.
61. Krishnan VB, Law AJ, Kenwright DN. Spinal extradural angiolipoma. Aust $N Z$ J Surg. Mar 1996;66(3):194-196.

62. O'Donovan NA, Naik K, Maloney WJ, Llewellyn CG. Spinal angiolipoma mimicking extradural lipomatosis. Can Assoc Radiol J. Feb 1996;47(1):51-53.

63. Provenzale JM, McLendon RE. Spinal angiolipomas: MR features. AJNR Am J Neuroradiol. Apr 1996;17(4):713-719.

64. Trabulo A, Cerqueira L, Monteiro J, Roque P, Reis FC, Coelho MR. Spinal angiolipomas revisited: two case reports. Acta Neurochir (Wien).

1996;138(11):1311-1319.

65. Boockvar JA, Black K, Malik S, Stanek A, Tracey KJ. Subacute paraparesis induced by venous thrombosis of a spinal angiolipoma: a case report. Spine (Phila Pa 1976). Oct 1 1997;22(19):2304-2308. 66. Shuangshoti S, Lerdlum S. Concurrent occurrence of solitary spinal epidural osteochondroma and angiolipoma. Clin Neuropathol. Mar-Apr 1997;16(2):107-110.

67. Sakaida H, Waga S, Kojima T, Kubo Y, Matsubara $\mathrm{T}$, Yamamoto J. Thoracic spinal angiomyolipoma with extracanal extension to the thoracic cavity. A case report. Spine (Phila Pa 1976). Feb 1 1998;23(3):391-394.

68. El Abbadi N, El Mostarchid B, Bellakhdar F. Thoracic epidural spinal angiolipoma: case report. Pan Arab J Neurosurg. 1999;3:49-51.

69. Kujas M, Lopes M, Lalam TF, Fohanno D, Poirier J. Infiltrating extradural spinal angiolipoma. Clin Neuropathol. Mar-Apr 1999;18(2):93-98. 70. Labram EK, el-Shunnar K, Hilton DA, Robertson NJ. Revisited: spinal angiolipoma--three additional cases. Br J Neurosurg. Feb 1999;13(1):25-29. 71. Oge HK, Soylemezoglu F, Rousan N, Ozcan OE. Spinal angiolipoma: case report and review of literature. J Spinal Disord. Aug 1999;12(4):353-356. 72. Turgut M. Spinal angiolipomas: report of a case and review of the cases published since the discovery of the tumour in 1890. Br J Neurosurg. Feb 1999;13(1):30-40.

73. Akhaddar A, Gazzaz M, Derraz S, et al. [Spinal epidural angiolipomas: a rare cause of spinal cord compression. A report of 8 cases and review of the literature]. Neurochirurgie. Dec 2000;46(6):523-533. 
74. al-Anazi A, Ammar A, Shannon P, al-Mulhim F. Spinal extradural angiolipoma. Br J Neurosurg. Oct 2000;14(5):471-472.

75. Andaluz N, Balko G, Bui H, Zuccarello M. Angiolipomas of the central nervous system. JNeurooncol. Sep 2000;49(3):219-230.

76. Bailey D, Andrews J, Mazur E, Cleary J. Dorsal thoracic cord compression from a spinal angiolipoma: case report and brief comment. Conn Med. May 2000;64(5):267-269.

77. Amlashi SF, Morandi X, Chabert E, Riffaud L, Haegelen C, Rolland Y. Spinal epidural angiolipoma. J Neuroradiol. Dec 2001;28(4):253-256.

78. Fourney DR, Tong KA, Macaulay RJ, Griebel RW. Spinal angiolipoma. Can J Neurol Sci. Feb 2001;28(1):82-88.

79. Gelabert-Gonzalez M, Agulleiro-Diaz J, ReyesSantias RM. Spinal extradural angiolipoma, with a literature review. Childs Nerv Syst. Dec 2002;18(12):725-728.

80. Pinto-Rafael JI, Vazquez-Barquero A, MartinLaez R, et al. [Spinal angiolipoma: case report]. $\mathrm{Neu}$ rocirugia (Astur). Aug 2002;13(4):321-325.

81. Aversa do Souto A, Dominues FS, Chimelli L, Lemos AM. Lumbosacral angiolipoma: case report. Arq Neuropsiquiatr. 2003(61):269-273.

82. Rabin D, Hon BA, Pelz DM, Ang LC, Lee DH, Duggal N. Infiltrating spinal angiolipoma: a case report and review of the literature. J Spinal Disord Tech. Oct 2004;17(5):456-461.

83. Rocchi G, Caroli E, Frati A, Cimatti M, Savlati M. Lumbar spinal angiolipomas: report of two cases and review of the literature. Spinal Cord. May 2004;42(5):313-316.

84. Samdani AF, Garonzik IM, Jallo G, Eberhart CG, Zahos P. Spinal angiolipoma: case report and review of the literature. Acta Neurochir (Wien). Mar 2004;146(3):299-302; discussion 302.

85. Cubillos A, Acevedo H, Castillo M, et al. Spinal extradural angiolipoma: report of one case presenting during pregnancy. Rev Chil Neurocirug.

2005(24):42-46.

86. Petrella G, Tamburrini G, Lauriola L, Di Rocco C. Spinal epidural angiolipoma complicated by an intratumoral abscess. Case report. J Neurosurg. Aug 2005;103(2 Suppl):166-169.

87. Dogan S, Arslan E, Sahin S, Aksoy K, Aker S.
Lumbar spinal extradural angiolipomas. Two case reports. Neurol Med Chir (Tokyo). Mar 2006;46(3):157-160.

88. Konya D, Ozgen S, Kurtkaya O, Pamir NM. Lumbar spinal angiolipoma: case report and review of the literature. Eur Spine J. Jun 2006;15(6):1025-1028.

89. Guzey FK, Bas NS, Ozkan N, Karabulut C, Bas SC, Turgut H. Lumbar extradural infiltrating angiolipoma: a case report and review of 17 previously reported cases with infiltrating spinal angiolipomas.

Spine J. Nov-Dec 2007;7(6):739-744.

90. Farooq MU, Samaraweera R, Heilman T, Chang HT. Spinal cavernous angiolipoma. Arch Neurol. Jul 2008;65(7):981-982.

91. Hungs M, Pare LS. Spinal angiolipoma: case report and literature review. J Spinal Cord Med. 2008;31(3):315-318.

92. Nanassis K, Tsitsopoulos $\mathrm{P}$, Marinopoulos D, Mintelis A, Tsitsopoulos P. Lumbar spinal epidural angiolipoma. J Clin Neurosci. Apr

2008;15(4):460-463.

93. Park JH, Jeon SR, Rhim SC, Roh SW. Lumbar spinal extradural angiolipoma: case report and review of the literature. J Korean Neurosurg Soc. Oct 2008;44(4):265-267.

94. Yen HL, Tsai SC, Liu SM. Infiltrating spinal angiolipoma. J Clin Neurosci. Oct 2008;15(10):1170-1173.

95. Dufrenot L, Pele E, Cursolle JC, Coindre JM, Lepreux S. [Spinal epidural angiolipoma: a case report]. Ann Pathol. Feb 2010;30(1):30-32.

96. Sankaran V, Carey M, Shad A. Traumatic bleeding of spinal angiolipoma presenting with subacute paraparesis--a case report and histopathological aspects. Br J Neurosurg. Dec 2010;24(6):714-715. 97. Chotai S, Hur JS, Moon HJ, Kwon TH, Park YK, Kim JH. Spinal angiolipoma--case report. Neurol Med Chir (Tokyo). 2011;51(7):539-542.

98. Diyora B, Nayak N, Kukreja S, Kamble H, Sharma A. Thoracic epidural angiolipoma with bilateral multilevel extraspinal extensions: a rare entity. Neurol India. Jan-Feb 2011;59(1):134-136.

99. Haji FA, Patel YK, Ang LC, Megyesi JF. A case of mistaken identity: spinal epidural angiolipoma. Can J Neurol Sci. Mar 2011;38(2):357-359.

100. Ghanta RK, Koti K, Dandamudi S. Spinal 
epidural angiolipoma: A rare cause of spinal cord compression. J Neurosci Rural Pract. Sep

2012;3(3):341-343.

101. Han SR, Yee GT, Choi CY, Lee CH. Infiltrating spinal angiolipoma. J Korean Neurosurg Soc. Aug 2012;52(2):161-163.

102. Fujiwara $\mathrm{H}$, Kaito $\mathrm{T}$, Takenaka $\mathrm{S}$, Makino $\mathrm{T}$, Yonenobu K. Thoracic spinal epidural angiolipoma: report of two cases and review of the literature. Turk Neurosurg. 2013;23(2):271-277.

103. da Costa MD, Paz Dde A, Rodrigues TP, et al. Hemorrhagic onset of spinal angiolipoma. J Neurosurg Spine. Dec 2014;21(6):913-915.

104. Nakao Y, Shimokawa N, Tsukazaki Y, Terada A, Nakajo K, Fu Y. Radical excision combined with instrumented fixation in the management of thoracic epidural angiolipoma: a case report. J Med Case Rep. 2014;8:377.

105. Si Y, Wang Z, Pan Y, Lin G, Yu T. Spinal angiolipoma: etiology, imaging findings, classification, treatment, and prognosis. Eur Spine J. Feb 2014;23(2):417-425.

106. Wang B, Yang Z, Yang J, Wang G, Xu Y, Liu P. Spinal angiolipoma: experience of twelve patients and literature. Neurol India. Jul-Aug 2014;62(4):367-370.

107. Nadi MM, Nadi AM, Zabara MY, Ahmad TM. Management of infiltrating spinal epidural angiolipoma. Neurosciences (Riyadh). Apr 2015;20(2):159-163. 108. Lin JJ, Lin F. Two entities in angiolipoma. A study of 459 cases of lipoma with review of literature on infiltrating angiolipoma. Cancer. Sep 1974;34(3):720-727.

109. Kitagawa $Y$, Miyamoto M, Konno S, et al. Subcutaneous angiolipoma: magnetic resonance imaging features with histological correlation. J Nippon Med Sch. 2014;81(5):313-319.

110. $\mathrm{Hu} \mathrm{S}, \mathrm{Hu} \mathrm{CH}, \mathrm{Hu} \mathrm{XY}$, et al. MRI features of spinal epidural angiolipomas. Korean J Radiol. Sep-
Oct 2013;14(5):810-817.

111. Garg A, Gupta V, Gaikwad S, et al. Spinal angiolipoma: report of three cases and review of MRI features. Australas Radiol. Mar 2002;46(1):84-90.

112. Klisch J, Spreer J, Bloss HG, Baborie A, Hubbe U. Radiological and histological findings in spinal intramedullary angiolipoma. Neuroradiology. Aug 1999;41(8):584-587.

113. Maggi G, Aliberti F, Colucci MR, Petrone G, Dorato P, De Giorgi AM. Spinal intramedullary angiolipoma. Childs Nerv Syst. Jun 1996;12(6):346-349.

114. Palkovic S, Wassmann H, Bonse R, Kashab M. Angiolipoma of the spinal cord. Magnetic resonance imaging and microsurgical management. Surg Neurol. Mar 1988;29(3):243-245.

115. Prasad GL, Sinha S. Spinal intradural subpial angiolipoma: Case report and review of literature. Surg Neurol Int. 2014;5:164.

116. Preul MC, Leblanc R, Tampieri D, Robitaille Y, Pokrupa R. Spinal angiolipomas. Report of three cases. J Neurosurg. Feb 1993;78(2):280-286.

\section{Disclosures}

The authors have no conflict of interests or funding sources to declare.

\section{Corresponding Author}

Enrique Caro-Osorio, M.D. Neurology and Neurosurgery Institute, Centro Médico Hospital Zambrano Hellion, TecSalud, Batallón de San Patricio \#112, 8th Floor, Monterrey, México 66278. ecaro@itesm.mx.

Published 2 December 2015.

This manuscript is generously published free of charge by ISASS, the International Society for the Advancement of Spine Surgery. Copyright $\odot 2015$ ISASS. To see more or order reprints or permissions, see http://ijssurgery.com. 